\title{
SI Correction
}

\section{MICROBIOLOGY}

Correction to Supporting Information for "Unveiling the biosynthetic puzzle of destruxins in Metarhizium species," by Bing Wang, Qianjin Kang, Yuzhen Lu, Linquan Bai, and Chengshu Wang, which appeared in issue 4, January 24, 2012, of Proc
Natl Acad Sci USA (109:1287-1292; first published January 9, 2012; 10.1073/pnas.1115983109).

The authors note that Fig. S1 appeared incorrectly. The SI has been corrected online.

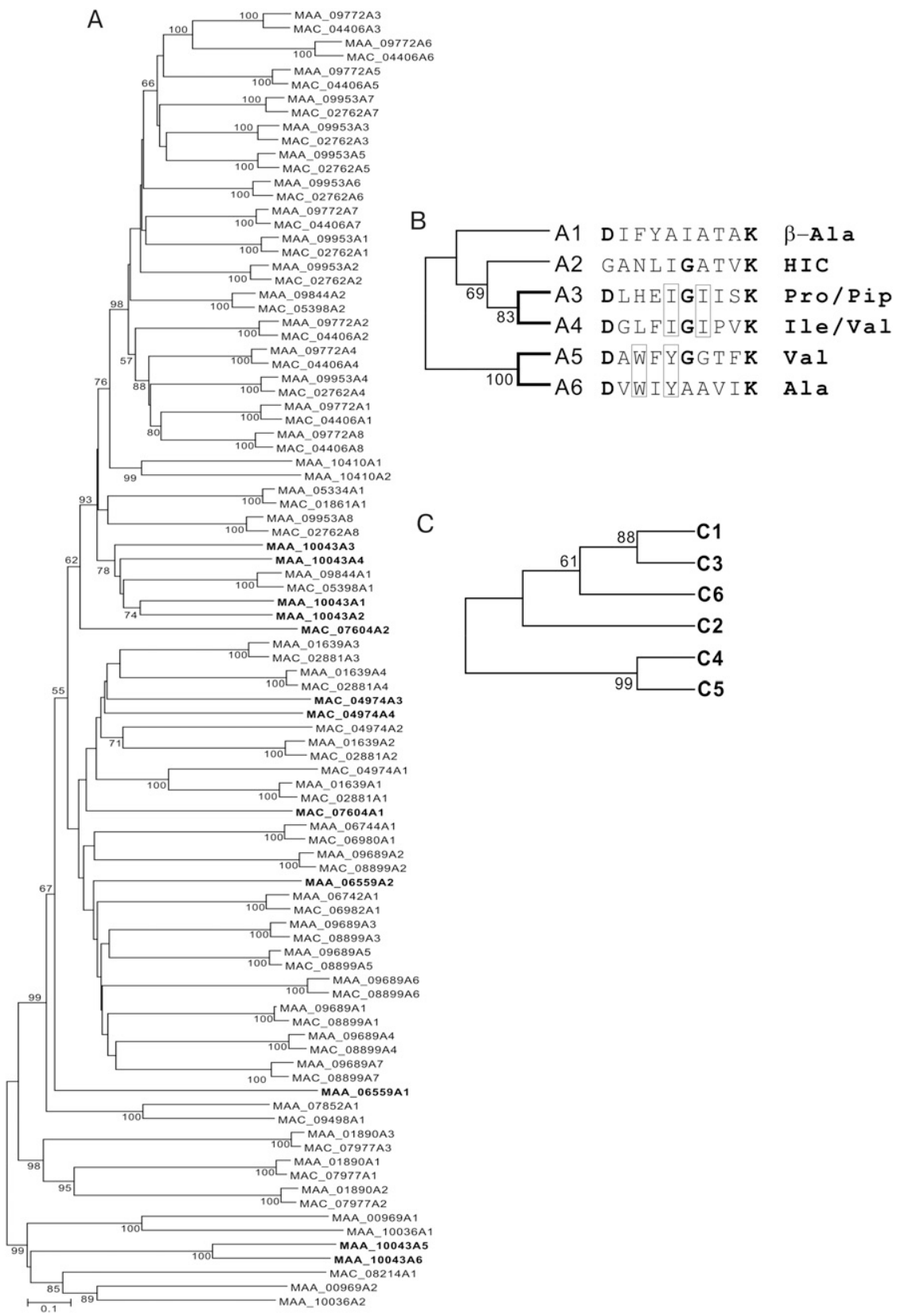

Fig. S1. Phylogenetic analysis. (A) Analysis of $M$. robertsii (loci tagged as MAA) and M. acridum (loci tagged as MAC) NRPSs based on their adenlylation domain sequences. The domains highlighted in bold indicate that the corresponding NRPS gene is present in one species but absent in the other. (B) Phylogenetic and signature analysis of DtxS1 A domains. (C) Analysis of DtxS1 C domains. 